\title{
Editorial
}

\section{Natural Products for Infectious Diseases}

\author{
Kang-Ju Kim, ${ }^{1}$ Xiangqian Liu, ${ }^{2}$ Takashi Komabayashi, ${ }^{3}$ Seung-Il Jeong, ${ }^{4}$ and Serkan Selli ${ }^{5}$ \\ ${ }^{1}$ Wonkwang University School of Dentistry, Iksan, Republic of Korea \\ ${ }^{2}$ Hunan University of Chinese Medicine School of Pharmacy, Changsha, China \\ ${ }^{3}$ University of New England College of Dental Medicine, Portland, ME, USA \\ ${ }^{4}$ Jeonju AgroBio-Materials Institute, Jeonju, Republic of Korea \\ ${ }^{5}$ University of Cukurova Faculty of Agriculture, Adana, Turkey \\ Correspondence should be addressed to Kang-Ju Kim; kjkimom@wku.ac.kr
}

Received 17 July 2016; Accepted 17 July 2016

Copyright (c) 2016 Kang-Ju Kim et al. This is an open access article distributed under the Creative Commons Attribution License, which permits unrestricted use, distribution, and reproduction in any medium, provided the original work is properly cited.

Infectious diseases have represented a threat to human lives since the beginning of human existence. Many infectious diseases have been conquered through the discovery of antibiotics and antiviral agents. However, the antibiotic-resistant strains and mutant microorganisms that are now emerging are more powerful than the existing ones. In addition, some existing microorganisms have developed resistance to antibiotics, leading to infections that are more difficult to treat. Moreover, microbial biofilms cannot be treated by antibiotics and can cause chronic infections. Infectious diseases continue to pose a threat to humans, and continued efforts are needed to develop effective treatments.

In recent times, natural products have been as widely used as chemical drugs against clinical diseases. Most chemical drugs that are widely used today were isolated from natural products, and thus natural products will continue to be important raw materials for the development of new drugs. However, since natural products are the byproducts of empirical medicine, they lack scientific validation. Currently, various scientific experiments are being conducted to fill this gap by evaluating the efficacy of natural product.

This special issue includes 7 research articles and 1 review article addressing the efficacies of natural products for treating infectious diseases, such as infection by multidrugresistant bacteria, viral influenza, coccidiosis, leishmaniasis, infectious septic shock, and biofilm formation. These articles represent pharmacological activity tests, investigation of action mechanisms of natural products, clinical trials with scientific statistical analyses, and phytochemical analyses of bioactive components in medicinal plants, which are important for scientific validation of the use of natural products in alternative and complimentary medicine.

\section{Acknowledgments}

We express our great gratitude to all authors for their contributions and reviewers for their great help. We convey our sincere thanks to the Editorial Board for their approval on this topic and continuous support of successful publication of this special issue. The Lead Guest Editor would like to thank the Guest Editors for their enthusiastic assistance. We hope this special issue will bring readers a useful academic reference in their research.

Kang-Ju Kim

Xiangqian Liu

Takashi Komabayashi Seung-Il Jeong Serkan Selli 


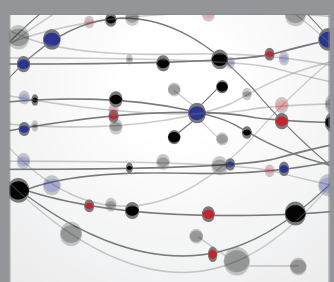

The Scientific World Journal
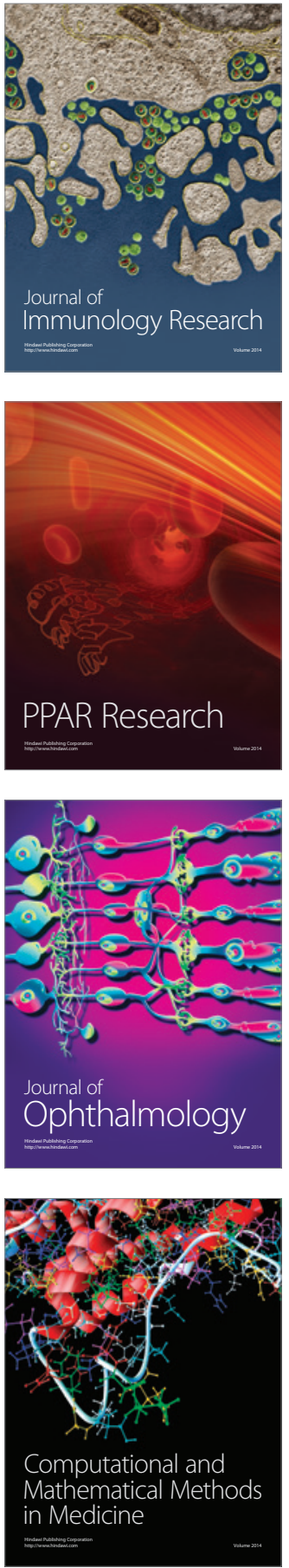

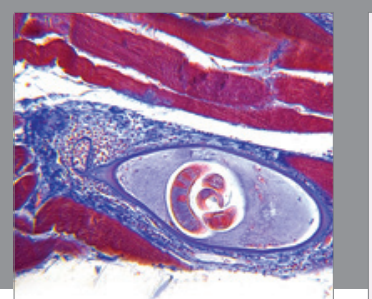

Gastroenterology Research and Practice

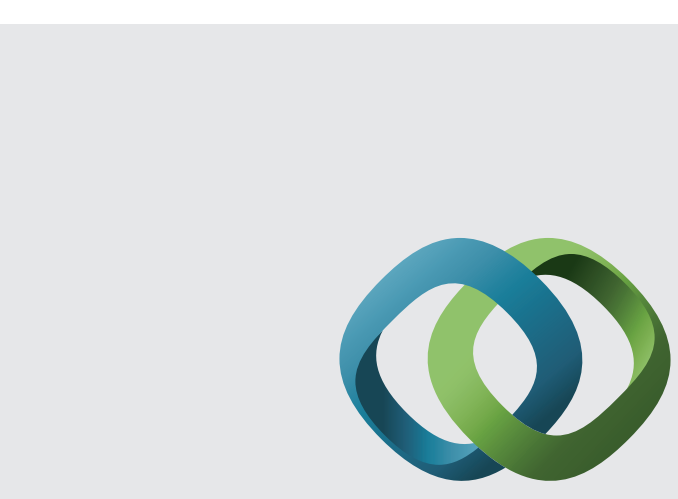

\section{Hindawi}

Submit your manuscripts at

http://www.hindawi.com
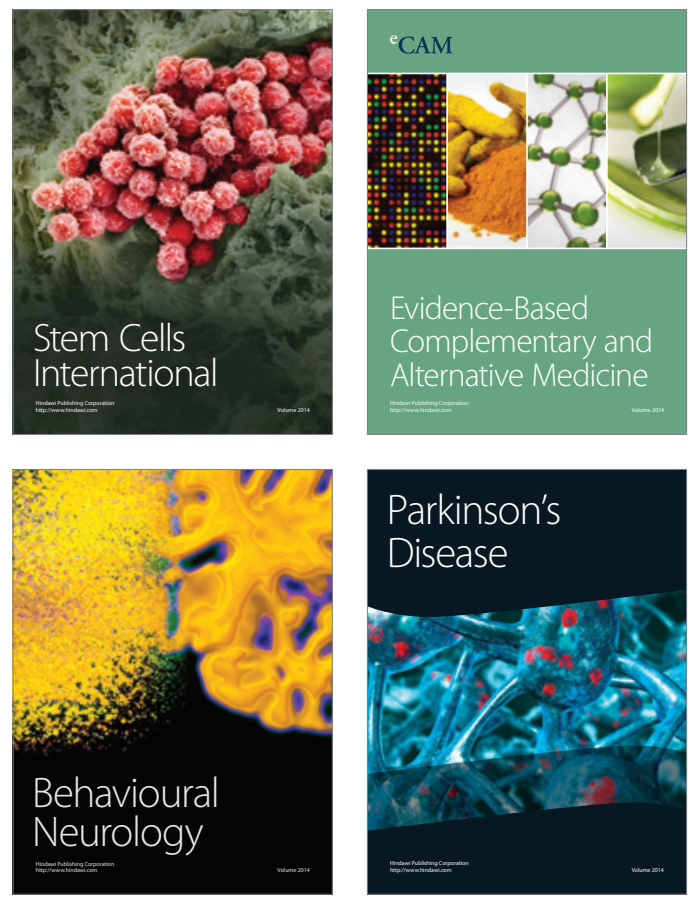
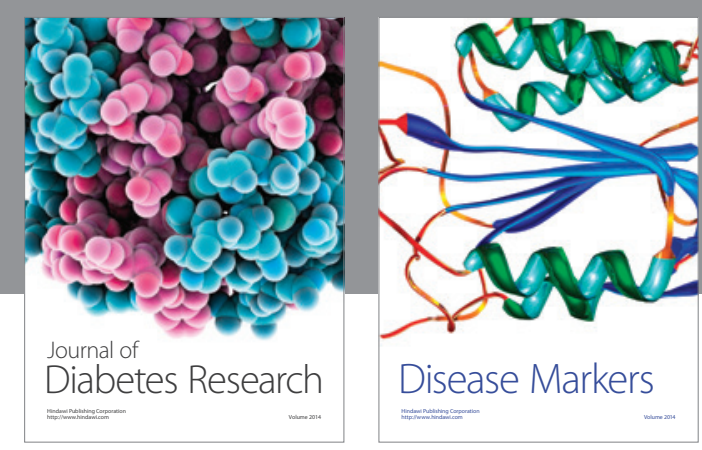

Disease Markers
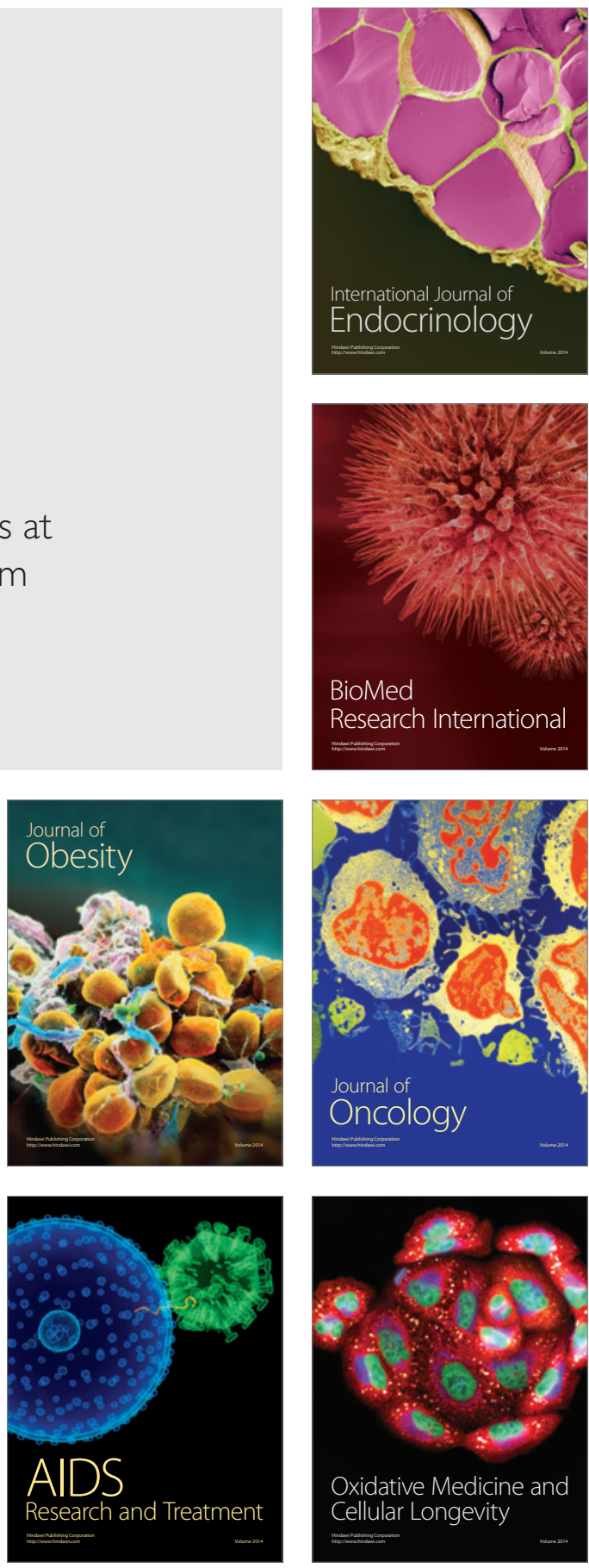